\title{
Caratteristiche della miastenia grave associata con l'autoimmunità tiroidea a Taiwan
}

\author{
Michele Marinò
}

Pubblicato online: 11 marzo 2014

C Springer International Publishing AG 2014

\section{Commento a:}

Clinical features of myasthenia gravis patients with autoimmune thyroid disease in Taiwan.

Y.L. Chen, J.H. Yeh, H.C. Chiu.

Acta Neurol Scand 2013, 127:170-174

In questo rimarchevole studio, condotto su di una casistica estremamente ampia, veniva esaminata la frequenza di autoimmunità tiroidea in pazienti con miastenia grave e venivano valutate le caratteristiche della miastenia stessa in relazione all'associazione o meno con l'autoimmunità tiroidea.

Tra il 1999 e il 2009 venivano studiati 1482 pazienti con miastenia grave. Di questi, $121(8,2 \%)$ presentavano un'associazione con malattie autoimmuni della tiroide conclamate (5,7\% morbo di Basedow e 1,1\% tiroidite autoimmune) o con la positività degli anticorpi anti-tiroide circolanti $(1,4 \%)$. I pazienti con associata autoimmunità tiroidea presentavano una maggior frequenza di miastenia oculare rispetto ai pazienti che non presentavano tale associazione. Questi ultimi presentavano più spesso la forma generalizzata di miastenia grave. Inoltre, la frequenza di timoma e quella degli autoanticorpi anti-recettore dell' acetilcolina circolanti erano significativamente più basse nei pazienti con miastenia oculare, che invece presentavano più spesso un'iperplasia timica.
Tali dati confermano in pieno quelli di uno studio precedente condotto nel nostro Centro oltre 15 anni or sono, su una casistica di 129 pazienti [1], e sostengono con forza la precedente conclusione che l'associazione tra autoimmunità tiroidea e miastenia grave è tipica delle forme più lievi di miastenia, quelle a esclusivo interessamento oculare, spesso senza timoma e sieronegative (autoanticorpi anti-recettore dell' acetilcolina circolanti assenti), la cui gestione terapeutica è estremamente più semplice. Queste osservazioni supportano l'ipotesi che la miastenia oculare e la miastenia generalizzata siano malattie diverse, con un differente spettro di malattie autoimmuni associate. La peculiare associazione tra miastenia oculare e tireopatie autoimmuni potrebbe riflettere meccanismi patogenetici comuni e/o una particolare predisposizione genetica.

\section{Bibliografia}

1. Marinò M, Ricciardi R, Pinchera A et al (1997) Mild clinical expression of myasthenia gravis associated with autoimmune thyroid diseases. J Clin Endocrinol Metab 82:438-443

\footnotetext{
M. Marinò $(\varangle)$

U.O. Endocrinologia 1, Università di Pisa, Pisa, Italia

e-mail: michele.marino@med.unipi.it
} 